\title{
Resveratrol attenuates inflammatory hyperalgesia by inhibiting glial activation in mice spinal cords
}

\author{
LIN-LIN WANG $^{1,2}$, DONG-LING SHI ${ }^{1,2}$, HUI-YAO GU ${ }^{3}$, MING-ZHI ZHENG ${ }^{4}$, \\ JUE $\mathrm{HU}^{4}$, XING-HUI SONG ${ }^{5}$, YUE-LIANG SHEN ${ }^{3}$ and YING-YING CHEN ${ }^{3}$ \\ ${ }^{1}$ Department of Physiology; ${ }^{2}$ Center for Stem Cell and Tissue Engineering; \\ ${ }^{3}$ Department of Pathology and Pathophysiology, Zhejiang University School of Medicine, Hangzhou, Zhejiang 310058; \\ ${ }^{4}$ Department of Pharmacology, Zhejiang Medical College, Hangzhou, Zhejiang 310053; \\ ${ }^{5}$ Core Facilities, Department of Basic Medical Sciences, Zhejiang University School of Medicine, \\ Hangzhou, Zhejiang 310058, P.R. China
}

Received April 30, 2015; Accepted March 4, 2016

DOI: $10.3892 / \mathrm{mmr} .2016 .5027$

\begin{abstract}
The present study aimed to investigate the effect of resveratrol on inflammatory pain. Mice were injected intraperitoneally with lipopolysaccharide (LPS) for 5 consecutive days to induce subacute systemic inflammation. Acetic acid-induced writhing tests and tail-flick tests were performed following the final LPS injection. Glial fibrillary acidic protein (GFAP; an astrocyte-specific activation marker), ionized calcium binding adapter molecule 1 (Iba-1; a microglia-specific activation marker) and sirtuin 1 (SIRT1) protein expression levels were detected using immunohistochemistry analysis or western blotting. Following administration of LPS for 5 days, the number of writhes increased and the tail-flick latency decreased. Resveratrol (10 or $20 \mathrm{mg} / \mathrm{kg}$ ) partly inhibited LPS-induced hyperalgesia and prevented the increase in tumor necrosis factor- $\alpha$ and interleukin 6 levels induced by LPS. LPS injection reduced the SIRT1 protein expression and increased the number of GFAP-positive and Iba-1-positive cells in the spinal cord. Resveratrol increased the SIRT1 protein expression levels and decreased the number of GFAP-positive and Iba-1-positive cells in LPS-treated mice. The protective effect of resveratrol was partly blocked by a selective SIRT1 inhibitor, EX-257. Results from the present study suggest that subacute treatment with LPS induced the activation of glial cells and hyperalgesia. Resveratrol was demonstrated to inhibit the activation of glial cells and attenuate inflammatory hyperalgesia in a SIRT1-dependent manner.
\end{abstract}

Correspondence to: Dr Ying-Ying Chen, Department of Pathology and Pathophysiology, Zhejiang University School of Medicine, 866 Yu-Hang-Tang Road, Hangzhou, Zhejiang 310058, P.R. China E-mail: bchenyy@zju.edu.cn

Key words: lipopolysaccharide, inflammation, pain, glial activation, sirtuin 1

\section{Introduction}

Pain facilitation has been reported to induce numerous nervous system-mediated sickness responses (1). Inflammation is crucial in the development and maintenance of persistent pain and inflammatory mediators are often altered regionally in acute pain (2); however, chronic pain patients have demonstrated a significant increase in pro-inflammatory cytokines, including tumor necrosis factor- $\alpha$ (TNF- $\alpha$ ), interleukin (IL)-1 $\beta$, IL-2, and IL-6 in plasma, which is associated with increased pain intensity $(3,4)$. Prolonged upregulation of inflammatory mediators in the injury epicenter, and regions above and below the lesion may be involved in chronic neuropathic pain in spinal cord injury (5). The systemic inflammatory response is also associated with pain and other symptoms in a cohort of cancer patients (6). Thus, anti-inflammatory intervention may be a useful therapeutic strategy in the management of pathological pain.

Resveratrol (3,5,4'-trihydroxy-trans-stilbene) is a non-flavonoid polyphenol present in grapes and red wine, which has been demonstrated to elicit a broad spectrum of biological responses in vitro and in vivo, including anticarcinogenic effects and neuroprotective and cardioprotective activities $(7,8)$. Previous studies have indicated that resveratrol is a potential therapeutic agent to attenuate bone cancer pain (9) and diabetic neuropathic pain (10). Injection of resveratrol into the cerebral ventricles also exerts evident central antalgic effects (11). Resveratrol has been demonstrated to interact with multiple molecular targets, a number of which are associated with inflammation and immunity (12). Thus, the current study hypothesized that resveratrol may also attenuate systemic inflammation-induced pain. The aim of the present study was to investigate the effect of resveratrol on inflammatory pain induced by the bacterial endotoxin, lipopolysaccharide (LPS), and investigate its underlying molecular mechanism.

\section{Materials and methods}

Reagents. LPS (from Escherichia coli 055:B5), resveratrol, sodium pentobarbital and EX-257 were purchased from 
Sigma-Aldrich (St. Louis, MO, USA). Dimethyl sulfoxide (DMSO), acetic acid, paraformaldehyde, $0.1 \mathrm{M}$ phosphate buffered saline (PBS; pH 7.4), paraffin, xylene, ethanol, hematoxylin and 3,3-diaminobenzidine were obtained from Sinopharm Chemical Reagent Co., Ltd. (Shanghai, China). Bovine serum albumin and 1\% Triton X-100 were obtained from Shanghai Sangon Biological Engineering Technology \& Services Co., Ltd. (Shanghai, China). Radioimmunoprecipitation lysis buffer, citrate buffer and BeyoECL Plus kit were purchased from Beyotime Institute of Biotechnology (Haimen, China). Primary antibodies for glial fibrillary acidic protein (GFAP; mouse monoclonal; sc-33673), sirtuin 1 (SIRT1; rabbit monoclonal; sc-15404), and $\beta$-actin (mouse monoclonal; sc-47778) were obtained from Santa Cruz Biotechnology, Inc. (Dallas, TX, USA). Primary antibodies for ionized calcium binding adapter molecule 1 (Iba-1; rabbit polyclonal; 019-19741) were purchased from Wako Pure Chemical Industries, Ltd. (Osaka, Japan). Biotin-labeled goat anti-rabbit IgG (A0277) and biotin-labeled goat anti-mouse $\operatorname{IgG}$ (A0286) secondary antibodies used in immunohistochemistry were obtained from Beyotime Institute of Biotechnology. Goat anti-rabbit $\operatorname{IgG}$ (7074) and horse anti-mouse $\operatorname{IgG}$ (7074) used in western blotting were obtained from Cell Signaling Technology, Inc. (Danvers, MA, USA). The enzyme-linked immunosorbent assay (ELISA) kits for TNF- $\alpha$ and IL-6 were obtained from R\&D Systems, Inc. (Minneapolis, MN, USA).

Mice. A total of 120 male ICR mice (age, 8-10 weeks; weight, 22-24 g) were obtained from the Experimental Animal Center of Zhejiang University (Hangzhou, China). Five mice per cage were housed in transparent plastic cages in controlled conditions at $20-24^{\circ} \mathrm{C}$ with $40-60 \%$ humidity and a $12 \mathrm{~h} \mathrm{light/dark}$ cycle. All mice were allowed ad libitum access to water and food. All experiments were conducted in accordance with the Guide for the Care and Use of Laboratory Animals published by National Institutes of Health (Bethesda, MD, USA) (13). All experimental protocols were approved by the Ethics Committee on Animal Experimentation of Zhejiang University.

Mice were divided into the following groups $(n=12)$ : i) Control group, which received the same volume of $0.1 \%$ DMSO vehicle diluted in saline $(0.1 \mathrm{ml} / 10 \mathrm{~g}$ body weight); ii) LPS group, which received an intraperitoneal (i.p.) injection of LPS $(0.25,0.5$, or $1 \mathrm{mg} / \mathrm{kg})$ for 5 consecutive days; iii) LPS + resveratrol group, which received an i.p. injection with resveratrol $(5,10$ or $20 \mathrm{mg} / \mathrm{kg}) 30 \mathrm{~min}$ prior to administration of LPS (1 mg/kg); iv) resveratrol group, which received an i.p. injection of resveratrol $(20 \mathrm{mg} / \mathrm{kg})$ for 5 consecutive days; v) LPS + resveratrol + EX-257 group, which received EX-257 $(2 \mathrm{mg} / \mathrm{kg})$ by i.p. injection $30 \mathrm{~min}$ prior to resveratrol $(20 \mathrm{mg} / \mathrm{kg})$ administration, which was followed by injection of LPS; and vi) EX-257 group, which received an i.p. injection of EX-257 (2 mg/kg). Behavioral assessments, histology and molecular biology analysis were performed $6 \mathrm{~h}$ after the final LPS injection of the experimental groups, or after the vehicle injection on the control group at the same time point. Resveratrol and EX-257 were dissolved in DMSO and diluted in saline. The concentration of DMSO was $<0.1 \%$, which exerted no marked physiological action or pharmacological anti-inflammatory effect.
Acetic acid-induced writhing test. The mice received an i.p. injection of $0.7 \%$ acetic acid $(0.1 \mathrm{ml} / 10 \mathrm{~g})$ and were then placed into separate plastic animal cages. The number of writhes was counted for 20 min after the i.p. injection according to the method described by Zhao et al (14). Writhing was defined as the contraction of abdominal muscles, which were accompanied by the stretching of the hind limbs.

Tail-fick test. To examine thermal hyperalgesia, the tail-flick test was employed according to the method described by Sora et al (15). Briefly, mice were restrained in conical polypropylene tubes with an opening, and their tails were immersed $\sim 2 \mathrm{~cm}$ into a $50 \pm 0.2^{\circ} \mathrm{C}$ water bath. The period from the immersion to the removal of the whole tail from the water was recorded as tail-flick latency.

Measurement of cytokines. Blood samples $(1 \mathrm{ml})$ were collected in test tubes containing ethylenediaminetetraacetic acid $(1 \mathrm{mg} / \mathrm{ml})$ and centrifuged immediately at $1,000 \mathrm{x} \mathrm{g}$ for $10 \mathrm{~min}$ at room temperature. The plasma was stored at $-80^{\circ} \mathrm{C}$ for analysis for cytokines. The plasma TNF- $\alpha$ and IL-6 levels were measured using ELISA kits according to manufacturer's protocols.

Immunohistochemistry. Mice were anesthetized with sodium pentobarbital $(60 \mathrm{mg} / \mathrm{kg}$, i.p.) and perfused with PBS via the ascending aorta to remove blood in the tissue prior to the fix-perfusion of $4 \%$ paraformaldehyde in $0.1 \mathrm{M}$ PBS (pH 7.4). The mice were then sacrificed by exsanguination. Spinal cord segments (lumbar (L)4-L5) were removed, post-fixed in the same fixative for $72 \mathrm{~h}$, and then embedded in paraffin. For immunohistochemistry, each specimen was cut into $5-\mu \mathrm{m}$ sections. The sections were deparaffinized in glass centrifuge tubes with two $10 \mathrm{ml}$ changes of xylene each $10 \mathrm{~min}$. Then, sections were rehydrated at room temperature in a sequence of decreasing concentrations of ethanol by 2 min incubations in $10 \mathrm{ml}$ of the following: $100 \%$ Ethanol 2 times, $90 \%$ ethanol 2 times, $70 \%$ ethanol and 50\% ethanol. After pretreatment with citrate buffer to enhance immunoreactivity, the sections were blocked in 5\% bovine serum albumin for $1 \mathrm{~h}$. The sections were incubated with anti-GFAP antibody (1:500) or anti-Iba-1 (1:500) overnight at $4^{\circ} \mathrm{C}$. After washing with PBS 3 times, the sections were incubated with biotin-conjugated secondary antibodies (1:200) for $1 \mathrm{~h}$ at room temperature. Sections were counterstained with hematoxylin to label nuclei. The staining was visualized using 3,3-diaminobenzidine, and observed with a BX51 microscope (Olympus Corporation, Tokyo, Japan). To quantify GFAP-positive and Iba-1-positive cells, images were captured of the dorsal horn of the spinal cord (magnification, $x 40$ ). Immunoreactive astrocytes and microglial cells with clearly identifiable nuclei were counted manually by an investigator who was blinded to the treatment and experienced in the field.

Western blotting. The spinal cords segments (dorsal part of L4-L5) were collected and homogenized in radioimmunoprecipitation lysis buffer containing $1 \%$ Triton X-100. Following centrifugation at $10,000 \mathrm{x}$ g at $4^{\circ} \mathrm{C}$ for $30 \mathrm{~min}$, the supernatant was collected and frozen at $-80^{\circ} \mathrm{C}$ until use. Lysates from the spinal cord were mixed with sodium dodecyl sulfate 


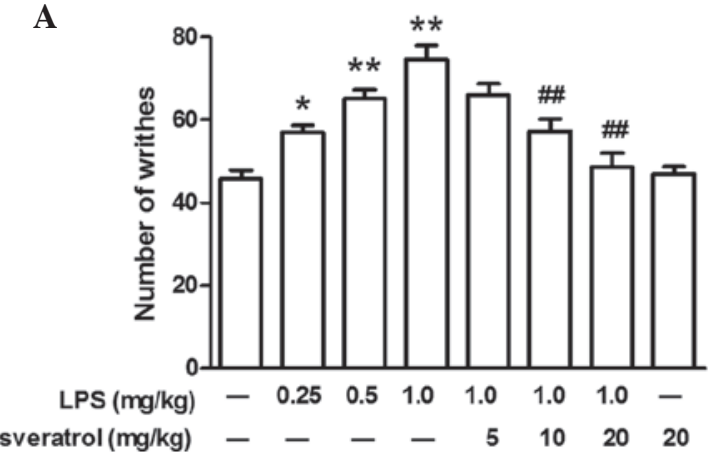

B

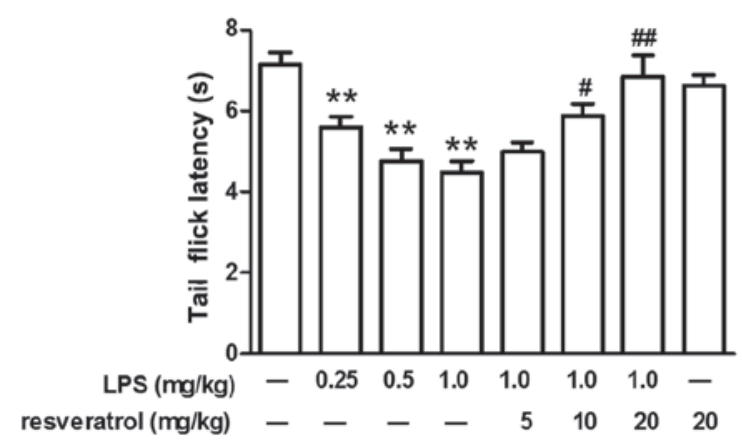

Figure 1. Effect of resveratrol on LPS-induced hyperalgesia in mice. (A) Number of writhes was obtained from the acetic acid-induced writhing test. (B) Latency was recorded in the tail-flick test. Data are presented as the mean \pm standard error of the mean $(\mathrm{n}=12)$. ${ }^{*} \mathrm{P}<0.05,{ }^{* *} \mathrm{P}<0.01$ vs. the control group; ${ }^{\#} \mathrm{P}<0.05,{ }^{\#} \mathrm{P}<0.01$ vs. the LPS $(1.0 \mathrm{mg} / \mathrm{kg})$ group. LPS, lipopolysaccharide.

sample buffer, heated at $100^{\circ} \mathrm{C}$ for $5 \mathrm{~min}$ and separated on $10 \%$ SDS-PAGE gels at $200 \mathrm{~V}$ for 40 min prior to transfer to a nitrocellulose membrane. Following blocking for $1 \mathrm{~h}$ with $5 \%$ skim milk, the membrane was incubated with primary antibodies $(1: 1,000)$ at $4^{\circ} \mathrm{C}$ overnight, followed by incubation with HRP-conjugated secondary antibodies $(1: 2,000)$ for $45 \mathrm{~min}$ at room temperature. All reactions were detected by the enhanced chemiluminescence detection method. The enhanced chemiluminescence signal was captured using a G:BOX Chemi XR5 imaging system (Syngene, Cambridge, UK). The band density was analyzed with Quality One software (version 4.6.2; Bio-Rad Laboratories, Inc., Hercules, CA, USA) and normalized to $\beta$-actin.

Statistical analysis. Data were expressed as the mean \pm standard error of the mean and analyzed by one-way analysis of variance with a Bonferroni post hoc test as required using Prism 5.0 (GraphPad Software, Inc., La Jolla, CA). P<0.05 was considered to indicate a statistically significant difference.

\section{Results}

LPS increased the number of writhes and decreased the latency of tail flicks. LPS-induced hyperalgesia in mice was evaluated by the acetic acid-induced writhing test and tail-flick test. Following the administration of LPS $(0.25,0.5$, or $1.0 \mathrm{mg} / \mathrm{kg}$, i.p.) for 5 days, the number of writhes increased

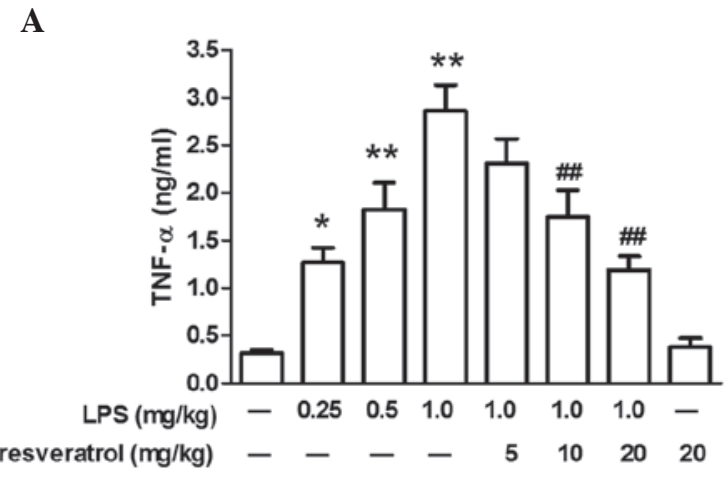

B

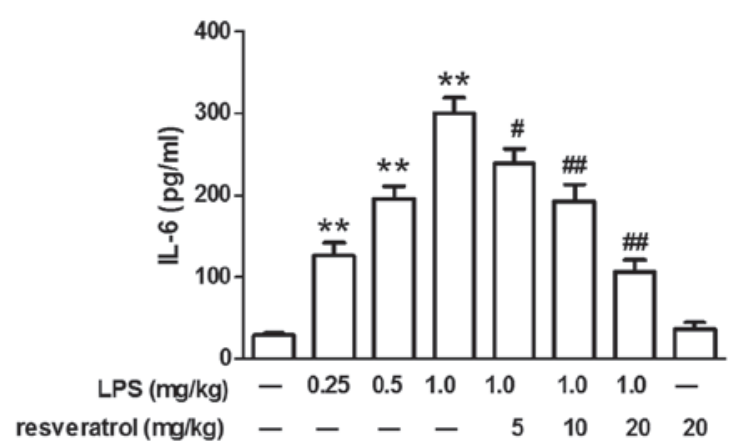

Figure 2. Effect of resveratrol on LPS-induced inflammation in mice. (A) Serum TNF- $\alpha$ level. (B) Serum IL-6 level. Data are presented as the mean \pm standard error of the mean $(n=12)$. ${ }^{*} \mathrm{P}<0.05,{ }^{* *} \mathrm{P}<0.01$ vs. the control group; ${ }^{\#} \mathrm{P}<0.05,{ }^{\# \#} \mathrm{P}<0.01$ vs. the LPS $(1.0 \mathrm{mg} / \mathrm{kg})$ group. LPS, lipopolysaccharide; TNF- $\alpha$, tumor necrosis factor- $\alpha$; IL-6, interleukin-6.

and the tail-flick latency decreased in a dose-dependent manner $(\mathrm{P}<0.05$; Fig. 1). As mice that were exposed to the highest concentration of LPS $(1.0 \mathrm{mg} / \mathrm{kg})$ exhibited marked hyperalgesia, this concentration of LPS was used for subsequent experiments. Administration of LPS once per day for 5 days did not alter body weight and although treatment with LPS induced slight behavioral alterations, one day after the final injection these behaviors, including grip tone, motor activity and swimming speed, were comparable with that of control group (data not shown).

Resveratrol partly inhibited LPS-induced hyperalgesia at 10 or $20 \mathrm{mg} / \mathrm{kg}$. Compared with the mice injected with LPS $(1.0 \mathrm{mg} / \mathrm{kg})$, resveratrol (10 or $20 \mathrm{mg} / \mathrm{kg}$ ) partly inhibited the LPS-induced hyperalgesia in the acetic acid-induced writhing test and tail-flick test, however, a lower dose of resveratrol $(5 \mathrm{mg} / \mathrm{kg}$ ) did not demonstrate any marked inhibitory effect on LPS-induced hyperalgesia. Injection of resveratrol alone did not influence the pain behavioral response in mice $(\mathrm{P}<0.05$; Fig. 1$)$.

Resveratrol blocked LPS-induced inflammation in mice. LPS injection significantly increased plasma TNF- $\alpha$ and IL-6 levels in mice $(\mathrm{P}<0.05)$. Resveratrol $(10$ or $20 \mathrm{mg} / \mathrm{kg})$ treatment reduced changes in IL- 6 or TNF- $\alpha$ expression levels induced by LPS ( $\mathrm{P}<0.01$; Fig. 2).

Resveratrol reduced the number of GFAP and Iba-1 positive cells in LPS treated mice. The astrocyte-specific activation 


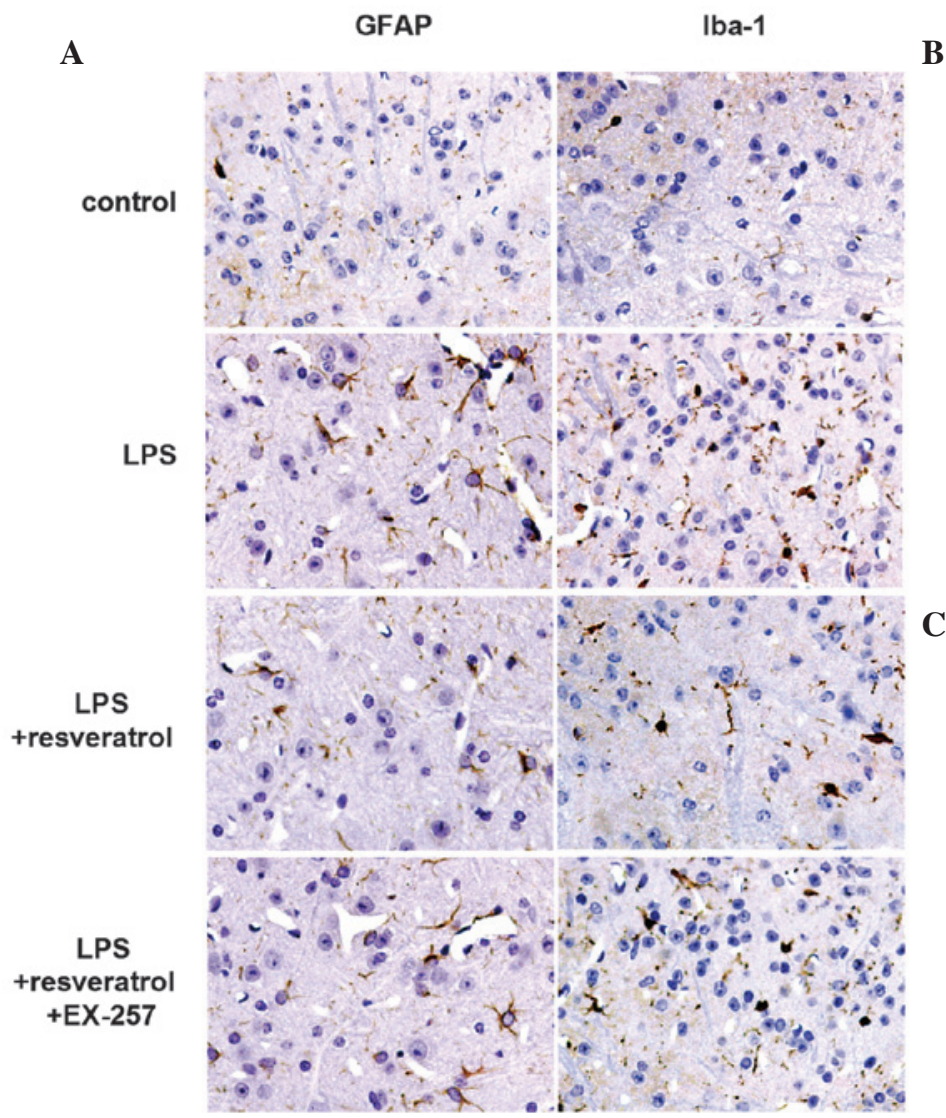

B

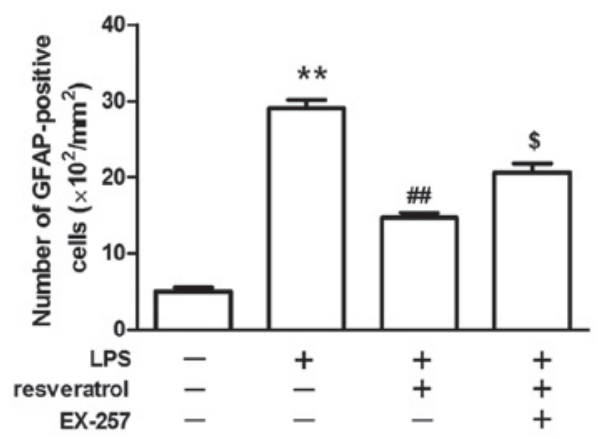

Figure 3. Immunohistochemical staining of GFAP and Iba-1 in the spinal cord of mice. (A) Representative microscopic field images (magnification, $\mathrm{x} 400$ ) of the dorsal horn of the spinal cord immunostained with GFAP and Iba-1. Number of (B) GFAP-positive and (C) Iba-1-positive glial cells. Data are presented as the mean \pm standard error of the mean $(\mathrm{n}=3)$. ${ }^{* *} \mathrm{P}<0.01$ vs. the control group; ${ }^{\# \#} \mathrm{P}<0.01$ vs. the LPS group; ${ }^{\$} \mathrm{P}<0.05,{ }^{\$ \$} \mathrm{P}<0.01$ vs. the LPS + resveratrol group. GFAP, glial fibrillary acidic protein; Iba-1, ionized calcium binding adapter molecule 1; LPS, lipopolysaccharide.

A

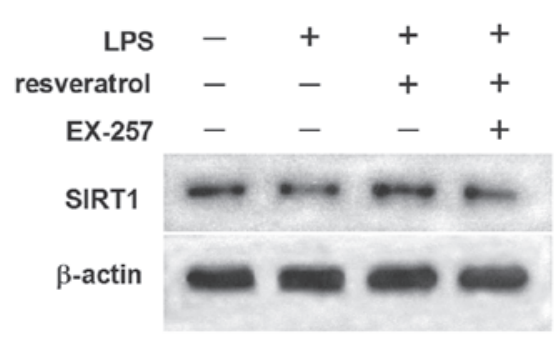

B

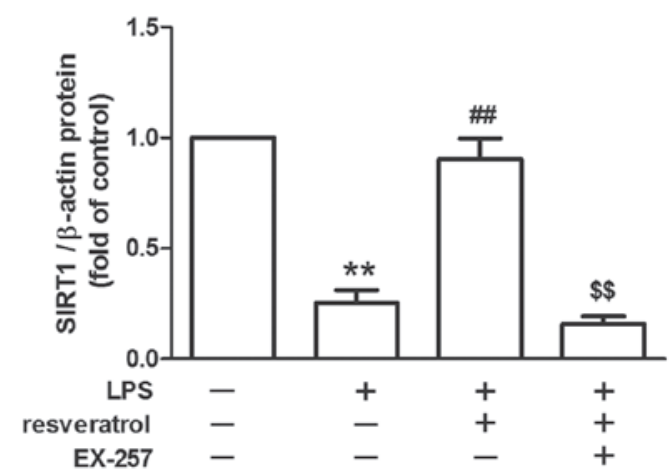

Figure 4. Western blotting analysis of SIRT1 protein expression in the dorsal spinal cord in mice. (A) Representative blots and (B) densitometric quantification of SIRT1 protein expression normalized to $\beta$-actin level. Data are presented as the mean \pm standard error of the mean $(n=3)$ and expressed as fold increase relative to the control value. ${ }^{* *} \mathrm{P}<0.01$ vs. the control group; ${ }^{\# \#} \mathrm{P}<0.01$ vs. the LPS group; ${ }^{\$ \$} \mathrm{P}<0.01$ vs. the LPS + resveratrol group. LPS, lipopolysaccharide; SIRT1, sirtuin 1. marker, GFAP and the microglia-specific activation marker, Iba-1 were detected by immunohistochemical staining. GFAP and Iba-1 staining was weak in the control group, however, the staining was markedly increased in the dorsal horns of the spinal cord following the injection of LPS. The number of GFAP-positive and Iba-1-positive cells in the spinal cord was markedly lower in the LPS + resveratrol group, when compared with the LPS treated group ( $\mathrm{P}<0.01$; Fig. 3$)$.

The inhibitory effect of resveratrol on LPS-induced hyperalgesia was blocked by a SIRT1 inhibitor. LPS injection reduced SIRT1 protein expression in the spinal cord. Resveratrol increased SIRT1 protein expression levels in the LPS treated mice ( $\mathrm{P}<0.01$; Fig. 4). EX-257, a selective SIRT1 inhibitor, blocked the resveratrol-induced analgesic effect $(\mathrm{P}<0.05$; Fig. 5), and EX-257 inhibited the anti-inflammatory effect exerted by resveratrol, and increased astrocyte and microglial activation, when compared with the LPS + resveratrol group $(\mathrm{P}<0.05$; Figs. 3 and 6$)$.

\section{Discussion}

In a number of chronic pain conditions, including neuropathic pain, cancer and obesity-associated pain, and other chronic inflammatory conditions, systemic inflammation is important in the pathophysiology of hyperalgesia, which may be mediated by the neural response in the spinal cord 
A

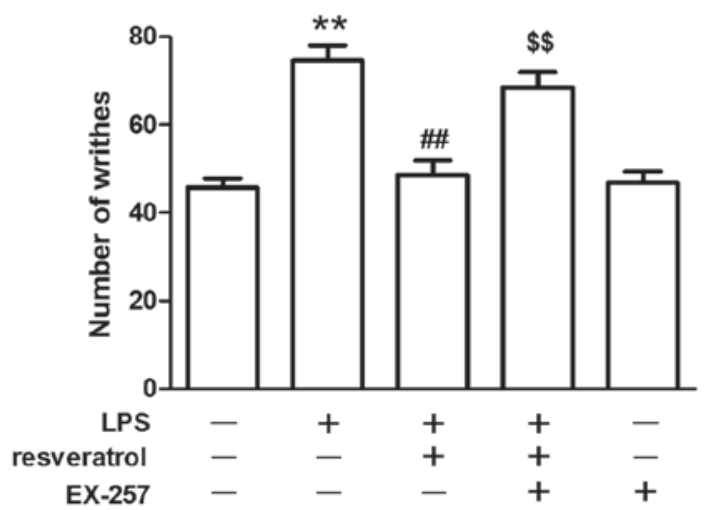

B

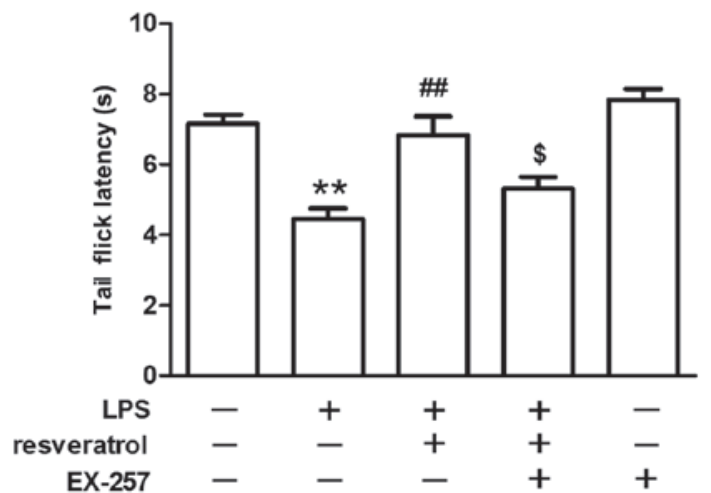

Figure 5. Effect of EX-257 on resveratrol-induced analgesia in mice. (A) Number of writhes observed in the acetic acid-induced writhing test. (B) Latency was recorded in the tail-flick test. Data are presented as the mean \pm standard error of the mean $(n=12) .{ }^{* *} \mathrm{P}<0.01$ vs. the control group ${ }^{\# \#} \mathrm{P}<0.01$ vs. the LPS group; ${ }^{\$} \mathrm{P}<0.05,{ }^{\$ \$} \mathrm{P}<0.01$ vs. the LPS + resveratrol group. LPS, lipopolysaccharide.

or brain $(5,6,16)$. In the present study, subacute i.p. injections of LPS were administered daily for five consecutive days to assess the hyperalgesia. Two different hyperalgesic tests were used, the acetic acid-induced writhing test and the tail flick test. In the acetic acid-induced abdominal writhing test (a visceral pain model), acetic acid nociceptive activity may be the result of a release of cytokines, including TNF- $\alpha$, IL-1 and IL-8 (17). In the present study, LPS increased the number of writhing events, which was consistent with the increased level of TNF- $\alpha$ and IL-6 in the plasma. However, the tail-flick tests were performed to assess whether resveratrol possesses central analgesic properties. In the tail-flick test, a thermal stimulus is focused on the tail skin of the animal to activate nociceptors in the superficial layers of the skin. The activation of peripheral nociceptors triggers a complex series of processes at the spinal level and results in the tail flick response. The latency of the tail-flick was decreased by LPS administration.

Glial cells, predominantly astrocytes and microglia, are important cell populations in the central nervous system (18). The glial cells are involved in maintaining homeostasis of the brain via regulation of $\mathrm{pH}$ and ionic balance, neurotransmitter uptake and degradation, and neuroinflammation modulation in physiological and pathophysiological conditions $(19,20)$. Increasing evidence indicates that the glial cells are crucial in the generation and maintenance of
A

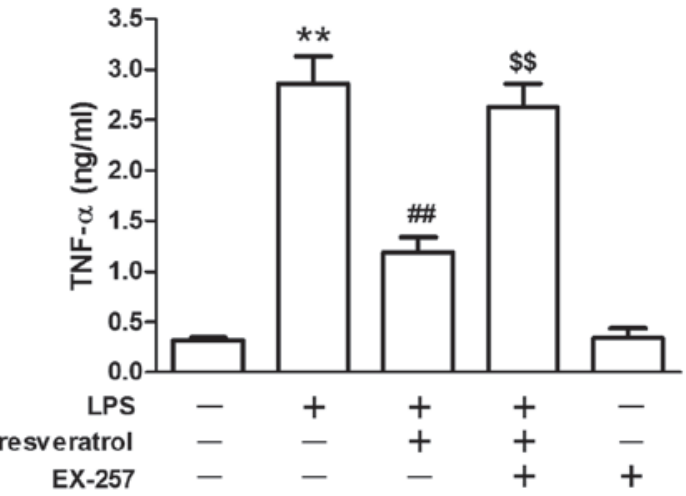

B

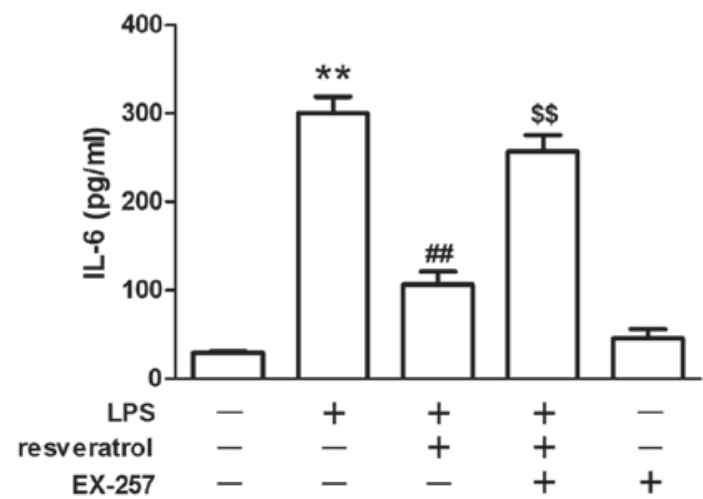

Figure 6. Effect of EX-257 on resveratrol-induced anti-inflammation in mice. (A) TNF- $\alpha$ levels and (B) IL-6 levels measured in the serum. Data are presented as the mean \pm standard error of the mean $(n=12) .{ }^{* *} \mathrm{P}<0.01$ vs. the control group; ${ }^{\# \#} \mathrm{P}<0.01$ vs. the LPS group; ${ }^{\$} \mathrm{P}<0.01$ vs. the LPS + resveratrol group. LPS, lipopolysaccharide; TNF- $\alpha$, tumor necrosis factor- $\alpha$; IL-6, interleukin-6.

chronic pain $(21,22)$. Ji et al $(23)$ reported that spinal astrocytic activation contributes to mechanical allodynia in a rat chemotherapy-induced neuropathic pain model. Activation of astrocytes in the anterior cingulate cortex has been demonstrated to be an affective component of pain in an inflammatory pain model (24). Astrocytic activation in the spinal cord also contributes to the development and maintenance of pain in rat models of chronic pancreatitis, chronic constriction of the sciatic nerve, and bone cancer (25-27). Furthermore, activated spinal microglia are key in neuropathic pain (28-30). GFAP is a specific astrocytic activation marker and Iba-1 is a specific microglial activation marker in the central nervous system (31). Although LPS may induce satellite glial cell activation in dorsal root ganglia (32), recent evidence suggests the importance of glial activation in the spinal cord $(33,34)$. In the present study, GFAP and Iba-1 expression levels were observed to be increased following administration of LPS for 5 days. The activation of glial cells may a result of the release of inflammatory cytokines in the rat dorsal horn by LPS $(35,36)$. Pharmacological inhibition of glial reactions may be a potential therapeutic strategy to treat inflammatory pain in animal models.

SIRT1 is a nicotinamide adenine dinucleotide-dependent histone deacetylase, which is involved in lifespan extension, age-associated disease delay, metabolism and apoptosis regulation (37-40). Recently, SIRT1 was demonstrated to regulate 
algesthesia. Upregulation of the expression of SIRT1 in the spinal dorsal horn reversed chronic morphine antinociceptive tolerance (41). Increased spinal SIRT1 expression attenuated mechanical allodynia and thermal hyperalgesia in a chronic constriction injury model $(42,43)$, and SIRT1 has also been observed to be crucial in inflammatory diseases, including obesity, type 2 diabetes mellitus, and atherosclerosis $(44,45)$. The present study showed that SIRT1 was downregulated in mice injected with LPS, suggesting that SIRT1 is important in modulating the development and progression of pain in subacute systemic inflammation. Thus, upregulation of SIRT1 appears to be a promising therapeutic strategy for systemic inflammation-induced pain.

SIRT1 has been reported to be one of the key targeted molecule of resveratrol and involved in its multiple biological effects (46-48). Resveratrol has potential anti-oxidative and anti-inflammatory properties and has been indicated to attenuate bone cancer pain (9). Chronic treatment with resveratrol for 4 weeks decreased the serum concentration of TNF- $\alpha$ and attenuated diabetic neuropathic pain (10). Systemic or spinal administration of resveratrol significantly suppresses morphine-induced microglial activation in the spinal cord (49). However, whether resveratrol relieved inflammation-induced pain remains to be elucidated. In the present study, it was observed that resveratrol inhibited the LPS-induced increase in serum inflammatory cytokines levels and attenuated inflammatory hyperalgesia in a dose-dependent manner. Furthermore, resveratrol inhibited the activation of glial cells in the dorsal horns of the spinal cord. The biological effect of resveratrol may be mediated by SIRT1-dependent (50) or SIRT1-independent (51) mechanisms, and phosphatidylinositol 3-kinase is essential for resveratrol-mediated expression of SIRT1 (50). The present study demonstrated that resveratrol increased the expression levels of SIRT1, and inhibition of SIRT1 expression by EX-257 blocked the resveratrol-induced analgesic effect.

A limitation of the present study is that the acute effect of resveratrol following the five treatments with LPS was not evaluated. Furthermore, whether the anti-hyperalgesia effect of resveratrol is more effective by intrathecal injection remains to be elucidated. However, the results of the present study suggest that resveratrol attenuates systemic inflammation-induced pain via upregulation of SIRT1. The protective effect of SIRT1 may occur via promotion of p65 deacetylation, regulation of mitogen activated protein kinase pathway, or inhibition of nuclear factor-kB activity $(52,53)$.

In conclusion, subacute administration of LPS induced the activation of glial cells and hyperalgesia. Resveratrol inhibited the activation of glial cells and attenuated inflammatory hyperalgesia in a SIRT1-dependent manner. The present study suggests that the beneficial role of resveratrol will help provide a potential therapeutic strategy in the treatment of pathological inflammatory pain.

\section{Acknowledgements}

The present study was supported by the Science and Technology Department of Zhejiang Province (grant no. 2014C37027 and 2014C37067) and the National Natural Science Foundation of China (grant no. 81371953).

\section{References}

1. Watkins LR and Maier SF: Immune regulation of central nervous system functions: From sickness responses to pathological pain. J Intern Med 257: 139-155, 2005.

2. Schinkel C, Gaertner A, Zaspel J, Zedler S, Faist E and Schuermann M: Inflammatory mediators are altered in the acute phase of posttraumatic complex regional pain syndrome. Clin J Pain 22: 235-239, 2006.

3. Koch A, Zacharowski K, Boehm O, Stevens M, Lipfert P, von Giesen HJ, Wolf A and Freynhagen R: Nitric oxide and pro-inflammatory cytokines correlate with pain intensity in chronic pain patients. Inflamm Res 56: 32-37, 2007.

4. Jiang YH, Peng CH, Liu HT and Kuo HC: Increased pro-inflammatory cytokines, $\mathrm{C}$-reactive protein and nerve growth factor expressions in serum of patients with interstitial cystitis/bladder pain syndrome. PLoS One 8: e76779, 2013.

5. Sandhir R, Gregory E, He YY and Berman NE: Upregulation of inflammatory mediators in a model of chronic pain after spinal cord injury. Neurochem Res 36: 856-862, 2011.

6. Laird BJ, McMillan DC, Fayers P, Fearon K, Kaasa S, Fallon MT and Klepstad P: The systemic inflammatory response and its relationship to pain and other symptoms in advanced cancer. Oncologist 18: 1050-1055, 2013.

7. Sun AY, Wang Q, Simonyi A and Sun GY: Resveratrol as a therapeutic agent for neurodegenerative diseases. Mol Neurobiol 41: 375-383, 2010.

8. Pangeni R, Sahni JK, Ali J, Sharma S and Baboota S: Resveratrol: Review on therapeutic potential and recent advances in drug delivery. Expert Opin Drug Deliv 11: 1285-1298, 2014.

9. Cheng W, Zhao Y, Liu H, Fan Q, Lu FF, Li J, Yin Q and Yan CD: Resveratrol attenuates bone cancer pain through the inhibition of spinal glial activation and CX3CR1 upregulation. Fundam Clin Pharmacol 28: 661-670, 2014

10. Sharma S, Kulkarni SK and Chopra K: Effect of resveratrol, a polyphenolic phytoalexin, on thermal hyperalgesia in a mouse model of diabetic neuropathic pain. Fundam Clin Pharmacol 21: 89-94, 2007.

11. Falchi M, Bertelli A, Galazzo R, Viganò P and Dib B: Central antalgic activity of resveratrol. Arch Ital Biol 148: 389-396, 2010.

12. Švajger U and Jeras M: Anti-inflammatory effects of resveratrol and its potential use in therapy of immune-mediated diseases. Int Rev Immunol 31: 202-222, 2012.

13. Institute of Laboratory Animal Resources (US). Committee on Care, Use of Laboratory Animals, and National Institutes of Health (US). Division of Research Resources: Guide for the care and use of laboratory animals. 8th edition. National Academies Press, Washington, DC, 2011.

14. Zhao H, Luo F, Li H, Zhang L, Yi Y and Wan J: Antinociceptive effect of tetrandrine on LPS-induced hyperalgesia via the inhibition of IKK $\beta$ phosphorylation and the $\mathrm{COX}-2 / \mathrm{PGE}_{2}$ pathway in mice. PLoS One 9: e94586, 2014.

15. Sora I, Takahashi N, Funada M, Ujike H, Revay RS, Donovan DM, Miner LL and Uhl GR: Opiate receptor knockout mice define mu receptor roles in endogenous nociceptive responses and morphine-induced analgesia. Proc Natl Acad Sci USA 94: 1544-1549, 1997.

16. Clark MR: Targeting systemic inflammation in patients with obesity-related pain: How best to prevent acute pain from becoming chronic? J Fam Pract 62 (9 Suppl CHPP): S3-S9, 2013.

17. Ribeiro RA, Vale ML, Thomazzi SM, Paschoalato AB, Poole S, Ferreira SH and CunhaFQ: Involvement of resident macrophages and mast cells in the writhing nociceptive response induced by zymosan and acetic acid in mice. Eur J Pharmacol 387: 111-118, 2000.

18. Jha MK, Seo M, Kim JH, Kim BG, Cho JY and Suk K: The secretome signature of reactive glial cells and its pathological implications. Biochim Biophys Acta 1834: 2418-2428, 2013.

19. Gwak YS, Kang J, Unabia GC and Hulsebosch CE: Spatial and temporal activation of spinal glial cells: Role of gliopathy in central neuropathic pain following spinal cord injury in rats. Exp Neurol 234: 362-372, 2012.

20. Parpura V, Heneka MT, Montana V, Oliet SH, Schousboe A, Haydon PG, Stout RF Jr, Spray DC, Reichenbach A, Pannicke T, et al: Glial cells in (patho)physiology. J Neurochem 121: 4-27, 2012.

21. Watkins LR and Maier SF: Beyond neurons: Evidence that immune and glial cells contribute to pathological pain states. Physiol Rev 82: 981-1011, 2002. 
22. Barragán-Iglesias P, Pineda-Farias JB, Cervantes-Durán C Bravo-Hernández M, Rocha-González HI, Murbartián J and Granados-Soto V: Role of spinal P2Y6 and P2Y11 receptors in neuropathic pain in rats: Possible involvement of glial cells. Mol Pain 10: 29, 2014.

23. Ji XT, Qian NS,Zhang T,Li JM,Li XK, Wang P,Zhao DS, Huang G, Zhang L, Fei Z, et al: Spinal astrocytic activation contributes to mechanical allodynia in a rat chemotherapy-induced neuropathic pain model. PLoS One 8: e60733, 2013.

24. Chen FL, Dong YL, Zhang ZJ, Cao DL, Xu J, Hui J, Zhu L and Gao YJ: Activation of astrocytes in the anterior cingulate cortex contributes to the affective component of pain in an inflammatory pain model. Brain Res Bull 87: 60-66, 2012

25. Feng QX, Wang W, Feng XY, Mei XP, Zhu C, Liu ZC, Li YQ, Dou KF and Zhao QC: Astrocytic activation in thoracic spinal cord contributes to persistent pain in rat model of chronic pancreatitis. Neuroscience 167: 501-509, 2010.

26. Cui J, He W, Yi B, Zhao H, Lu K, Ruan H and Ma D: mTOR pathway is involved in ADP-evoked astrocyte activation and ATP release in the spinal dorsal horn in a rat neuropathic pain model. Neuroscience 275: 395-403, 2014.

27. Shen W, Hu XM, Liu YN, Han Y, Chen LP, Wang CC and Song C: CXCL12 in astrocytes contributes to bone cancer pain through CXCR4-mediated neuronal sensitization and glial activation in rat spinal cord. J Neuroinflammation 11: 75, 2014

28. Vega-Avelaira D, Ballesteros JJ and López-García JA: Inflammation-induced hyperalgesia and spinal microglia reactivity in neonatal rats. Eur J Pain 17: 1180-1188, 2013

29. Xu ZZ, Berta T and Ji RR: Resolvin E1 inhibits neuropathic pain and spinal cord microglial activation following peripheral nerve injury. J Neuroimmune Pharmacol 8: 37-41, 2013.

30. Moss A, Beggs S, Vega-Avelaira D, Costigan M, Hathway GJ, Salter MW and Fitzgerald M: Spinal microglia and neuropathic pain in young rats. Pain 128: 215-224, 2007.

31. Guo J, Jia D, Jin B, Xu F, Yuan X and Shen H: Effects of glial cell line-derived neurotrophic factor intrathecal injection on spinal dorsal horn glial fibrillary acidic protein expression in a rat model of neuropathic pain. Int J Neurosci 122: 388-394, 2012.

32. Blum E, Procacci P, Conte V and Hanani M: Systemic inflammation alters satellite glial cell function and structure. A possible contribution to pain. Neuroscience 274: 209-217, 2014

33. Cao FL, Xu M, Wang Y, Gong KR and Zhang JT: Tanshinone IIA attenuates neuropathic pain via inhibiting glial activation and immune response. Pharmacol Biochem Behav 128: 1-7, 2015

34. Silva GD, Lopes PS, Fonoff ET and Pagano RL: The spinal anti-inflammatory mechanism of motor cortex stimulation: Cause of success and refractoriness in neuropathic pain? J Neuroinflammation 12: 10,2015.

35. Zhu MD, Zhao LX, Wang XT, Gao YJ and Zhang ZJ: Ligustilide inhibits microglia-mediated proinflammatory cytokines production and inflammatory pain. Brain Res Bull 109: 54-60, 2014.

36. Clark AK, Staniland AA, Marchand F, Kaan TK, McMahon SB and Malcangio M: P2X7-dependent release of interleukin-1beta and nociception in the spinal cord following lipopolysaccharide. J Neurosci 30: 573-582, 2010.

37. Luo XY, Qu SL, Tang ZH, Zhang Y, Liu MH, Peng J, Tang H, Yu KL, Zhang C, Ren Z and Zhang ZS: SIRT1 in cardiovascular aging. Clin Chim Acta 437: 106-114, 2014.
38. Herskovits AZ and Guarente L: SIRT1 in neurodevelopment and brain senescence. Neuron 81: 471-483, 2014.

39. Leibiger IB and Berggren PO: Sirt1: A metabolic master switch that modulates lifespan. Nat Med 12: 34-36, 2006.

40. Kalle AM, Mallika A, Badiger J, Alinakhi, Talukdar P and Sachchidanand: Inhibition of SIRT1 by a small molecule induces apoptosis in breast cancer cells. Biochem Biophys Res Commun 401: 13-19, 2010.

41. He X, Ou P, Wu K, Huang C, Wang Y, Yu Z and Guo Q: Resveratrol attenuates morphine antinociceptive tolerance via SIRT1 regulation in the rat spinal cord. Neurosci Lett 566: 55-60, 2014.

42. Yin Q, Lu FF, Zhao Y, Cheng MY, Fan Q, Cui J, Liu L, Cheng W and Yan CD: Resveratrol facilitates pain attenuation in a rat model of neuropathic pain through the activation of spinal Sirt1. Reg Anesth Pain Med 38: 93-99, 2013.

43. Shao H, Xue Q, Zhang F, Luo Y, Zhu H, Zhang X, Zhang H, Ding W and Yu B: Spinal SIRT1 activation attenuates neuropathic pain in mice. PLoS One 9: e100938, 2014.

44. Winnik S, Stein S and Matter CM: SIRT1-an anti-inflammatory pathway at the crossroads between metabolic disease and atherosclerosis. Curr Vasc Pharmacol 10: 693-696, 2012.

45. Xie J, Zhang X and Zhang L: Negative regulation of inflammation by SIRT1. Pharmacol Res 67: 60-67, 2013.

46. Joe IS, Jeong SG and Cho GW: Resveratrol-induced SIRT1 activation promotes neuronal differentiation of human bone marrow mesenchymal stem cells. Neurosci Lett 584: 97-102, 2015.

47. Li L, Sun Q, Li Y, Yang Y, Yang Y, Chang T, Man M and Zheng L: Overexpression of SIRT1 Induced by resveratrol and inhibitor of miR-204 suppresses activation and proliferation of microglia. J Mol Neurosci 56: 858-867, 2015.

48. Sin TK, Tam BT, Yung BY, Yip SP, Chan LW, Wong CS, Ying M, Rudd JA and Siu PM: Resveratrol protects against doxorubicin-induced cardiotoxicity in aged hearts through the SIRT1-USP7 axis. J Physiol 593: 1887-1899. 2015.

49. Han Y, Jiang C, Tang J, Wang C, Wu P, Zhang G, Liu W, Jamangulova N, Wu X and Song X: Resveratrol reduces morphine tolerance by inhibiting microglial activation via AMPK signalling. Eur J Pain 18: 1458-1470, 2014.

50. Zong Y, Sun L, Liu B, Deng YS, Zhan D, Chen YL, He Y, Liu J, Zhang ZJ, Sun J and Lu D: Resveratrol inhibits LPS-induced MAPKs activation via activation of the phosphatidylinositol 3-kinase pathway in murine RAW 264.7 macrophage cells. PLoS One 7: e44107, 2012

51. Centeno-Baez C, Dallaire P and Marette A: Resveratrol inhibition of inducible nitric oxide synthase in skeletal muscle involves AMPK but not SIRT1. Am J Physiol Endocrinol Metab 301: E922-E930, 2011.

52. Yang H, Zhang W, Pan H, Feldser HG, Lainez E, Miller C, Leung S, Zhong Z, Zhao H, Sweitzer S, et al: SIRT1 activators suppress inflammatory responses through promotion of $\mathrm{p} 65$ deacetylation and inhibition of NF-kappaB activity. PLoS One 7: e46364, 2012.

53. Park GJ, Kim YS, Kang KL, Bae SJ, Baek HS, Auh QS, Chun YH, Park BH and Kim EC: Effects of sirtuin 1 activation on nicotine and lipopolysaccharide-induced cytotoxicity and inflammatory cytokine production in human gingival fibroblasts. J Periodontal Res 48: 483-492, 2013. 